\title{
International High School Big Data Challenge 2018, New York Academy of Sciences
}

\section{Résumés du High Data Big Data Challenge 2018: Académie des sciences de New York}

Amanda Zheng, Ani Ter-Margaryan, Angela Wang, Archi Parekh, Arunima Sen, Catherine Aitken, Cindy Liu, Dhanush Adithyan, Erol Balkovic, Gaurang Bharti, Isaac Fung, Javier Arevalo, Johan Jeson, Kelly Kang, Kevin Clyde Luis Gerardo Nunez, Kitty Haque, Manaal Shah, Michael Geiss, Miu Sugaya, Moreno Mc. Manus, Mrunali Manjrekar, Perry Son, Rakshit Jain, Sachin Dangi, Sama Yassar, Tasnuba Sukanna, Vikram Mishra, Xiuyu Li

The New York Academy of Sciences' Big Data: Think Global, Act Local Challenge provided an opportunity for students to experience working with data analytics by combing scientific theories with statistics, programming and innovation. Students analyzed large data sets provided by the STEM Fellowship and other open access sets to reveal patterns and trends related to human behavior and interactions.

98 students participated from 29 countries who formed 26 teams that were overseen by 20 participating mentors. The teams were invited to address one of three issues: the correlation between food and climate change, renewable energy and sustainable infrastructure, or climate change and the economy. They investigated how big data can shed light on these issues and whether the connections made influence the future on both a government and individual level.

They were encouraged to use a variety of data analysis algorithms, including classification algorithms, regression, network analysis text analysis and association rules.

Each teams' solutions were judged on a variety of criteria which included: how innovative their solution was, if the concept was clear and concise, if the analysis showed potential to make a difference and how, , potential social impact, and if the experience was a collaborative endeavor.

The Big Data Challenge was sponsored by Medidata, Regeneron and S\&P Global.

New York Academy of Sciences

\section{Disclaimer}

These abstracts are provided for all student teams that have submitted project reports by May 28, 2018. The STEM Fellowship Journal editorial board has made every effort to ensure proof and English editing of these abstracts in a limited amount of time, and neither organization as a whole or any of its volunteer members can be held accountable for inaccuracies that may have occurred in the abstract publication. Abstracts are published in alphabetical order per abstract titles:

\section{Barley: The Impact of Climate Change on the Farming Process}

Archi Parekh, Arunima Sen, Dhanush Adithyan, Javier Arevalo, Kevin Clyde Moreno Mc. Manus

A possible increase of altered agricultural growing patterns has occurred due to the rising prevalence of climate change. Understanding its effects on staple crop yields is necessary to guarantee food security in the future. Barley, one of such crops, is classified as susceptible to the effects of changing climate and yet little research has been done to determine exactly how it has been impacted. The aim of this study was to quantify the effects of climate change on barley yields in the United States. The United Nations, National Oceanic and Atmospheric Administration, and the World Bank provided datasets for: barley yields, average temperature, precipitation index, Palmer Drought Severity Index (PDSI), Palmer Z Index (ZNDX), and carbon dioxide emissions from 1961 to 2016. The primary tool for statistical analysis was Microsoft Excel. In the analysis process, two independent sample t-tests assuming unequal variances disproved the null hypothesis, which stated that temperature, precipitation, PDSI, and ZNDX do not affect barley yield. Further correlation analyses showed that temperature and precipitation had a weak correlation with yield. PDSI and ZNDX showed no correlation with barley yields, while carbon dioxide emissions correlated strongly. The findings suggest a connection between barley yields and temperature, precipitation, and carbon dioxide emissions. Further analysis is necessary to uncover confounding factors affecting barley yields. Follow-up analysis would calculate the difference in barley yields in conditions 
with and without climate change factors to better quantify effects of climate change. The results of this study demonstrate the need for farmers to further consider climate change in the farming process.

\section{Consequences of Climate Change and Actions Tak- en to Fight it}

Luis Gerardo Nunez, Manaal Shah

Anthropogenic climate change is a threat to humanity. The aim of this investigation was to study the economic effects of government and private actions to fight climate change and determine if these actions are effective in their current form. To determine their effectiveness, we studied the relation between greenhouse gas emissions (methane and carbon dioxide) and the global temperature over a 30 -year period. We also investigated the global albedo rate and the emission of aerosols during the 21 st century. We studied the effects that certain governmental policies have had on GHG emissions and their projected impact to determine their success in reducing emissions. Over the last 35 years, there has been a 1000 - 3000 ppb increase in carbon dioxide emissions and a $0.25^{\circ}-1.25^{\circ} \mathrm{C}$ increase in global temperature. In addition to this, methane emissions in 2017 are lower compared to methane emissions in 1984. From the data gathered, it appears that the Pan-Canadian Approach to Pricing Carbon Pollution will produce better results than the projected outcome before the implementation of the policy. This indicates that current policies are better but still don't meet the requirements set in the Paris Agreements.

\section{Evaluating the Economic Impacts prompted by Differing Responses to Natural Disasters}

Kitty Haque, Michael Geiss, Mrunali Manjrekar, Perry Son

Rising temperatures have greatly contributed to variances in climate-based anomalies. This has led to variances in natural disasters that plague affected nations regularly. Across the United States, state-level governments have responded to different natural disasters including severe storms, hurricanes, and tornadoes through either budgetary allocations or requests to the federal government for aid. In other cases, states have had lackluster responses, which result in economic turmoil within local communities. The aim of our study is to evaluate the economic impacts brought on by different responses to natural disasters. A series of datasets from FEMA (Federal Emergency Management Agency) were utilized, where the amount of federal aid distributed was compared with the type of natural disaster, the state, and the level of preparedness based on budget. Microsoft Excel was utilized to conduct analysis. California, Texas, Puerto Rico, and the Virgin Islands were required the most considerable amount of federal assistance. Georgia, California, and Texas were found to be the least prepared for natural disasters through budgetary analysis. This offered an explanation to why the disasters were more damaging in those states. As a result, the respective local governments were required to search for more federal support than other states, suggesting that climate change put additional economic strain on the nation. To continue the study, investigations will continue regarding the economic strains posed by the cost of damage from natural disasters, then will shift to analyzing how economics influences climate change using chi-square tests to determine the extent of the two variables' relationship.

\section{Predicting Heat Waves Using Big Data}

Catherine Aitken, Erol Balkovic, Gaurang Bharti, Johan Jeson, Miu Sugaya, Vikram Mishra

Natural disasters have been responsible for millions of deaths and the destruction of urban lands worldwide. If predicted in advance, valuable lives and properties could be saved. Current algorithms for predicting natural disasters are based on human expertise, however, this results in varying predictions for the same data set. This project builds a machine learning algorithm to predict heat waves by studying the trends in weather and climate data. The weather patterns for each natural disaster is different, with the chosen disaster being the occurrence of heatwaves. The data set used is from Chihuahua, Mexico, due to it containing the highest number of heat waves recorded. During data preprocessing, missing values in the dataset were imputed from other days in the week. eatures that had too many missing values or were irrelevant to our prediction were dropped. Data balancing was essential since the number of days on which heatwaves did not occur was exponentially higher than the number of heatwaves recorded. A decision tree model was developed to classify whether a heatwave occurs on a given day with the variables used being temperature, dew point, precipitation, wind speed and visibility. With a test 
sample of $20 \%$ and a learning sample of $80 \%$, the algorithm was able to predict heatwave occurrences with an accuracy of $83 \%$. The algorithm learns from actual instances rather than from strict rules set by experts and has the potential to get better over time as it learns from more data. This algorithm can easily be applied for predicting other natural disasters such as droughts, hurricanes, cyclones, etc. and can save lives throughout the world by warning residents in advance and helping authorities take preventive measures.

\section{Renewable Energy Resource Consumption and Climate Change in the US}

Amanda Zheng, Ani Ter-Margaryan, Isaac Fung, Rakshit Jain, Xiuyu Li

Our planet is approximately 200 to 250 gigatonnes of carbon emissions away from a $1.5^{\circ} \mathrm{C}$ rise in global temperatures - the limit proposed by the Paris Climate Accord. This study analyses the effects of renewable energy resource consumption on climate change in the US to address the pressing issue of global warming. Our study first used a k-means classification algorithm to analyze the Berkleyearth Dataset, revealing a positive correlation between carbon emissions and rising global temperatures; the Earth will not be able to sustain a continued temperature increase such as the one predicted. The second data analysis, performed in MATLAB using the DSIRE Dataset, revealed that the total US consumption of renewable energy has increased from 1960 to 2015. The third data analysis, performed in MATLAB using the State Energy Data System Dataset, revealed that most states already obtain most of their energy from renewable sources, thus implementing new government policies will do little to increase the amount of renewable energy production. Our study concludes that the only viable solutions to global warming are to take preventative measures and to change the way the world uses energy to avoid further climate change impacts. This includes the introduction of policies in transport, urban planning, and the usage of more eco-friendly technologies that will reduce industrial smokestack emission and improve the efficiency of buildings, making cities more compact.

\section{The Environental Impacts of Livestock Farming}

Sama Yassar, Sachin Dangi, Cindy Liu, Tasnuba Sukanna, Kelly
Kang, Angela Wang

This study aimed to identify and analyze the correlation between livestock, greenhouse gas emissions, and climate change. Six of the most common livestock, cattle, buffalo, sheep, goat, pig, and chicken, were charted to reveal the amount of each greenhouse gas released because of their farming. We considered factors such as enteric fermentation, manure, and feed. The goal of this data research was to discover the most impactful livestock-emitted greenhouse gas and its direct source. As climate change becomes an increasingly more prevalent concern, it is important to heighten our awareness surrounding the production of food and the effect that it has on our climate. Following the initial charting, further focus was directed into the issue by narrowing our attention to the enteric fermentation of ruminant animals, specifically cattle. Our data revealed that greenhouse gas emissions resulted from beef cattle alone emit approximately 2495 million tonnes of carbon dioxide into the atmosphere annually. Dairy cattle account for approximately another 2128 million tonnes, followed by pigs, buffalo, chicken, and small ruminants at 668 million, 618 million, 612 million, and 474 million tonnes, respectively. These numbers point strongly to the trend that the farming of livestock has a direct proportional relationship to greenhouse gas levels in the atmosphere, and thus translates to the correlation between livestock greenhouse gas emissions and climate change. These findings present the cruciality of further research into techniques to reduce the environmental impacts of agriculture. 